\title{
Calibrant Delivery for Mass Spectrometry
}

\author{
Bradley B. Schneider and Thomas R. Covey \\ MDS SCIEX, Concord, Ontario, Canada
}

This article describes a means of sampling ions that are created at a location remote from the primary ion source used for mass spectral analysis. Such a source can be used for delivery of calibrant ions on demand. Calibrant ions are sprayed into an atmospheric pressure chamber, at a position substantially removed from the sampling inlet. A gas flow sweeps the calibrants towards the sampling inlet, and a new means for toggling the second ion beam into the instrument can be achieved with the use of a repelling field established by an electrode in front of the sampling inlet. The physical separation of two or more sources of ions eliminates detrimental interactions due to gas flows or fields. When using a nanoflow electrospray tip as the primary ion source, the potential applied to the tip completely repels calibrant ions and there is no compromise in terms of electrospray performance. When calibrant ions are desired, the potential applied to the nanoflow electrospray tip is lowered for a short period of time to allow calibrant ions to be sampled into the instrument, thus providing a means for external calibration that avoids the typical complications and compromises associated with dual spray sources. It is also possible to simultaneously sample ions from multiple ion beams if necessary for internal mass calibration purposes. This method of transporting additional ion beams to a sampling inlet can also be used with different types of atmospheric pressure sources such as AP MALDI, as well as sources configured to deliver ions of different polarity. (J Am Soc Mass Spectrom 2007, 18, 991-996) (C) 2007 American Society for Mass Spectrometry

A ccurate mass assignment provides improved identification confidence for mass spectrometry, and the degree to which it can be achieved varies with different mass analyzers. A number of calibrant introduction systems currently exist in the patent and general literature. The simplest approach involves using a single sprayer ESI system and either teeing calibrants in postcolumn or adding them to the LC mobile phase [1]. Under these conditions, the calibrant delivery can not be quickly turned on or off; consequently, the calibrants ions are always present in the mass spectra where they can overlap with the $\mathrm{m} / \mathrm{z}$ of ions of interest. Other drawbacks with these approaches are potential matrix effects, loss of chromatographic resolution due to dead-volumes inherent in nanoflow connections, and/or contamination of LC pumps. To avoid these problems, a number of different approaches involving multiple sprayers have been reported, as described in a recent tutorial [2]. This article describes a new approach for analysis of multiple ion beams that can provide a calibrant on-demand system with minimal detrimental effect on the analyte signal. Indexing is achieved by controlling the potential of the analytical source, with sampling on/off times about 40 to $100 \mathrm{~ms}$, and no measurable cross-talk between the sources.

Address reprint requests to Dr. B. B. Schneider, MDS SCIEX, 71 Four Valley, Drive, Concord, Ontario, Canada, L4K 4V8. E-mail: bradley.schneider@ sciex.com

\section{Experimental}

All samples were purchased from Sigma Chemical Co. (St. Louis, MO). Methanol and formic acid were purchased from Fischer Scientific (Nepean, ON, CA). Standards were prepared by dissolving the samples in solutions comprised of 50/50 methanol/water with $0.1 \%$ formic acid to various concentrations.

The mass spectrometer used for these experiments was a 4000 Q TRAP instrument (Applied Biosystems/ MDS SCIEX, Foster City, CA) equipped with a modified particle discriminator interface (PDI) [3]. All nanoflow electrospray experiments were carried out using the standard MicroIonSpray II assembly [4] located in close proximity to the inlet of the curtain plate. Tapered fused silica sprayers (New Objective Inc., Woburn, MA) were used with inner, outer, and tapered tip diameters of 20, 360 , and $10 \mu \mathrm{m}$, respectively, to limit the dead-volume after the conductive liquid junction. For some experiments, a second MicroIonSpray II was used to provide calibrant or reagent ions. The second sprayer was held in place with a ring stand, and the standard Gas 2 connection on the source was used to provide compressed air for the nebulizer in the additional sources. An additional Spellman (Hauppauge, NY) power supply provided the potential for the calibrant or reagent ion sprayers. Samples were delivered to the sprayers using syringe infusion 22 pumps (Harvard Apparatus, South Natick, MA).

MALDI experiments were conducted using a modified AP MALDI source (MassTech Inc., Columbia, MD) 


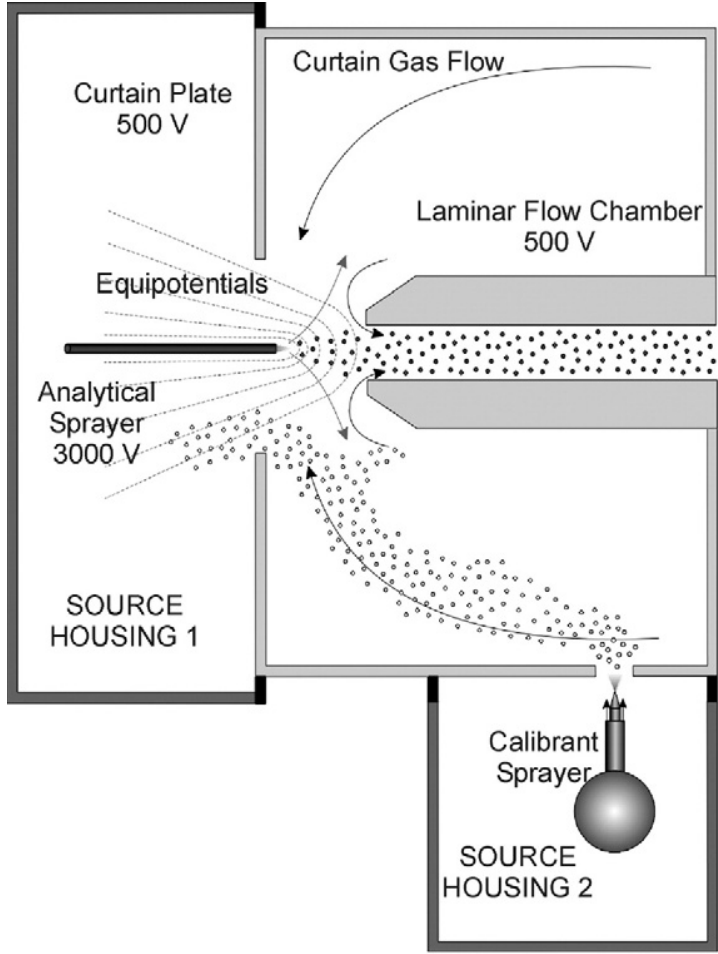

(a)

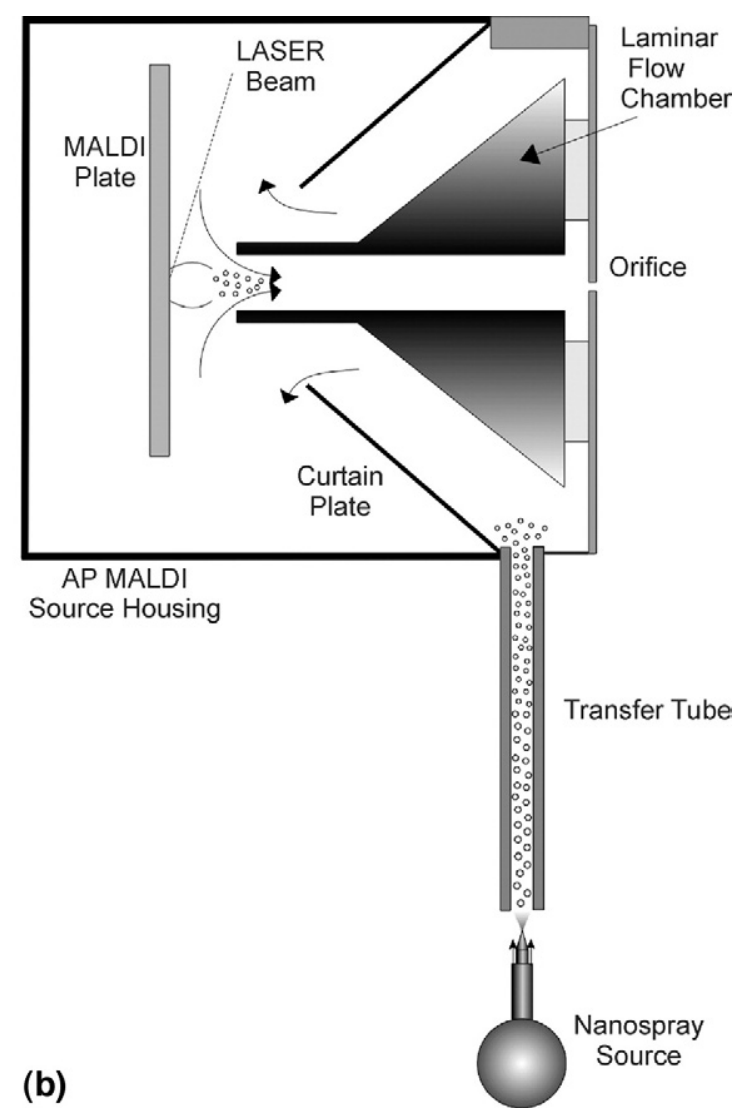

Figure 1. (a) Schematic of the indexed dual source system showing the field from the optimized analytical sprayer preventing calibrant ions from entering the sampling inlet. (b) Schematic of a dual source configuration including an AP MALDI source located in a first housing proximal to a sampling inlet and a nanoflow electrospray source located outside of the AP MALDI source housing. An additional sampling tube is included to transport ions from the remote electrospray source to a chamber located adjacent to the sampling inlet.

as described previously [5]. The system included a nitrogen laser and $100 \mu \mathrm{m}$ optical fiber to illuminate samples deposited on a stainless steel sample plate from Applied Biosystems (Foster City, CA). Samples were co-crystallized with $\alpha$-cyano-4-hydrocinnamic acid.

\section{Results}

An ideal mass calibrant delivery system for nanoflow ESI needs to provide calibrant ions while not affecting the performance or tuning of the primary analytical sprayer. One way to achieve this with dual electrospray sources is to space the sprayers a long distance from each other; however, mass spectrometers typically have a single inlet aperture such that wide spacing of the sprayers leads to reduced performance for one or both of the sprayers. Reduced performance is acceptable for a calibrant sprayer provided that the performance is stable and the analytical sprayer is unaffected. The system described in this paper is optimized to eliminate any performance compromise for an analytical source, positioning it in the optimum location in close proximity to the mass spectrometer inlet. An additional source is incorporated away from the MS inlet and bleeds ions into an atmospheric pressure chamber before the sampling inlet as shown in Figure 1a. A curtain plate establishes a sealed chamber before the sampling inlet of the mass spectrometer. Since the sampling inlet draws gas from this chamber into the instrument, ions present in the gas will be sampled, regardless of whether they are generated by a source located close to the sampling inlet (referred to as the analytical sprayer), or located distant to the inlet (referred to as a calibrant sprayer in Figure 1a). An auxiliary gas, such as a Curtain Gas flow or other independent gas stream will carry a portion of the calibrant ions and droplets to the sampling inlet. The potential applied to the analytical sprayer will establish a field between it and the inlet aperture, effectively repelling any calibrant ions and charged droplets of like charge converging on the inlet through the gas stream. When the potential applied to the analytical sprayer is lowered to approximately the same level as the potential applied to both the curtain plate and the sampling inlet, the region within the curtain chamber becomes essentially field free, and a portion of the calibrant ions and charged droplets are swept into the inlet along with a portion of the Curtain Gas flow. When the voltage on the analytical sprayer is 

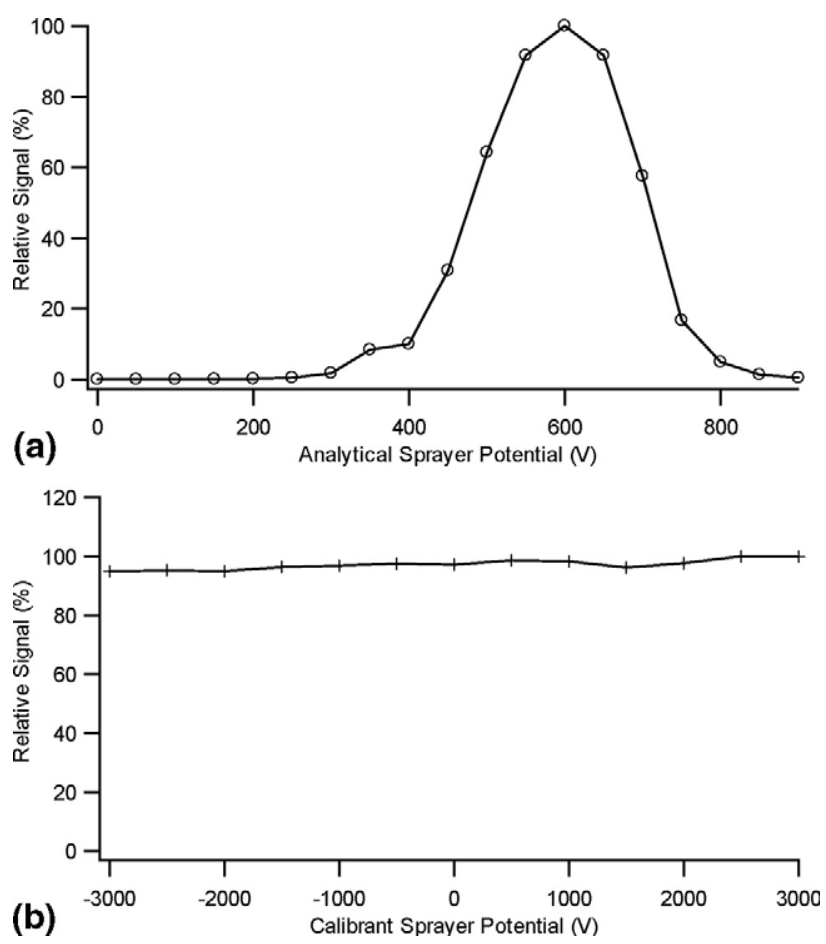

Figure 2. (a) MRM data for minoxidil calibrant ions showing the effect of the potential applied to the analytical sprayer on the ion count rate for calibrant ions. The MRM transition was $210 \rightarrow 193$, and the potential applied to the analytical sprayer varied from 0 to $900 \mathrm{~V}$ with a fixed calibrant sprayer potential of $3000 \mathrm{~V}$. (b) MRM data for reserpine ions from the analytical sprayer showing the effect of the potential applied to the calibrant sprayer on the ion count rate. The MRM transition was $609 \rightarrow 195$ and the calibrant source was positioned $\sim 3 \mathrm{~cm}$ to the right of the analytical sprayer, directly in front of a $2 \mathrm{~mm}$ aperture in the curtain plate.

lowered, ions are no longer produced but the calibrant ions and charged droplets are allowed to enter the sampling aperture. In this manner, one can synchronize the ion production from a particular source with the data acquisition system.

Figure 1a demonstrates an optimized configuration where the analytical sprayer is located in a source housing close to the sampling inlet and the calibrant sprayer is located in a different source housing substantially removed from the sampling inlet. The isolation of the two sources prevents effects such as ion beam deflection [6] and nebulizer gas interactions that commonly occur when operating with multiple high voltage pneumatically nebulized sources in close proximity. However, the principle of introducing calibrant ions sufficiently far from the sampling inlet so as to not affect the performance of an analytical sprayer can also be applied to the situation where multiple sprayers are located within a single source housing, provided that there are multiple apertures into the curtain chamber and the sprayers are positioned sufficiently far from each other to limit field or gas flow interactions.

Figure 2 shows an example of experimental data generated with dual nanoelectrospray sources illustrating the effects of the analytical sprayer potential on the signal for calibrant ions (Figure 2a) as well as the effects of the calibrant sprayer potential on the signal for ions from the analytical sprayer (Figure $2 b$ ). The curtain plate and inlet aperture were at $\sim 600 \mathrm{~V}$. When the potential applied to the analytical sprayer was within $\pm 100 \mathrm{~V}$ of this value, the field was sufficiently low to allow calibrant ions and charged droplets to enter the inlet aperture. With the standard operating potential of $\sim 3000 \mathrm{~V}$ applied to the analytical sprayer, no calibrant ions were observed in the instrument. Toggling the calibrant ion signal with the analytical sprayer voltage requires field penetration into the curtain chamber; therefore, the voltage range over which it is effective is proportional to the distance the analytical sprayer is from the sampling inlet. In Figure 2a, where the curtain plate and inlet voltage was $600 \mathrm{~V}$, the voltage range for the analytical sprayer to significantly attenuate the calibrant signal bracketed the inlet voltage by $\pm 200 \mathrm{~V}$. In this case, the sprayer was approximately flush with the curtain plate ( $3 \mathrm{~mm}$ from the heated inlet). Moving the analytical sprayer $1 \mathrm{~mm}$ outside of the curtain plate increased this range to $\pm 400 \mathrm{~V}$ and decreased it to \pm 50 $\mathrm{V}$ when the analytical sprayer was inserted through the curtain plate by $\sim 2 \mathrm{~mm}$, always centered on the potential applied to the curtain plate and heated inlet with this geometry.

Figure $2 b$ shows the MRM signal for reserpine ions generated by the analytical sprayer as the potential applied to the calibrant sprayer varied from $-3000 \mathrm{~V}$ to the standard operating potential of $\sim 3000 \mathrm{~V}$. The potential applied to the calibrant sprayer had no effect on the count rate for ions from the analytical sprayer, even when the calibrant sprayer polarity was switched to generate negative ions. The combined effects of the calibrant sprayer potential and the presence of calibrant ions and charged or neutral droplets within the curtain chamber had no effect on either the count rate for ions from the analytical sprayer or the tuning of the analytical sprayer (nebulizer gas flow, electrospray potential, or position). The calibrant ions were essentially invisible unless desired.

When using the configuration depicted in Figure 1, it is desirable to minimize the diameter of the calibrant introduction aperture through the curtain plate to minimize losses of Curtain Gas. Empirical experiments were done to optimize this aperture while operating the analytical sprayer at the optimal potential to allow calibrant ions through. A $4 \mathrm{~mm}$ aperture provided maximum signal and reducing it to $2 \mathrm{~mm}$ and finally 1 $\mathrm{mm}$ decreased the signal from a Glufibrinopeptide B solution by 30 and $85 \%$, respectively. Since the curtain plate aperture for the analytical sprayer is $10 \mathrm{~mm}$, an additional $2 \mathrm{~mm}$ calibrant aperture affects the total gas flow rate through the standard aperture by less than $5 \%$. A $2 \mathrm{~mm}$ aperture was thus chosen as the best compromise for minimizing Curtain Gas and calibrant ion losses. It should be pointed out that the calibrant nebulizer was orientated at an angle relative to the inlet aperture to minimize the disturbance of the gas flow 

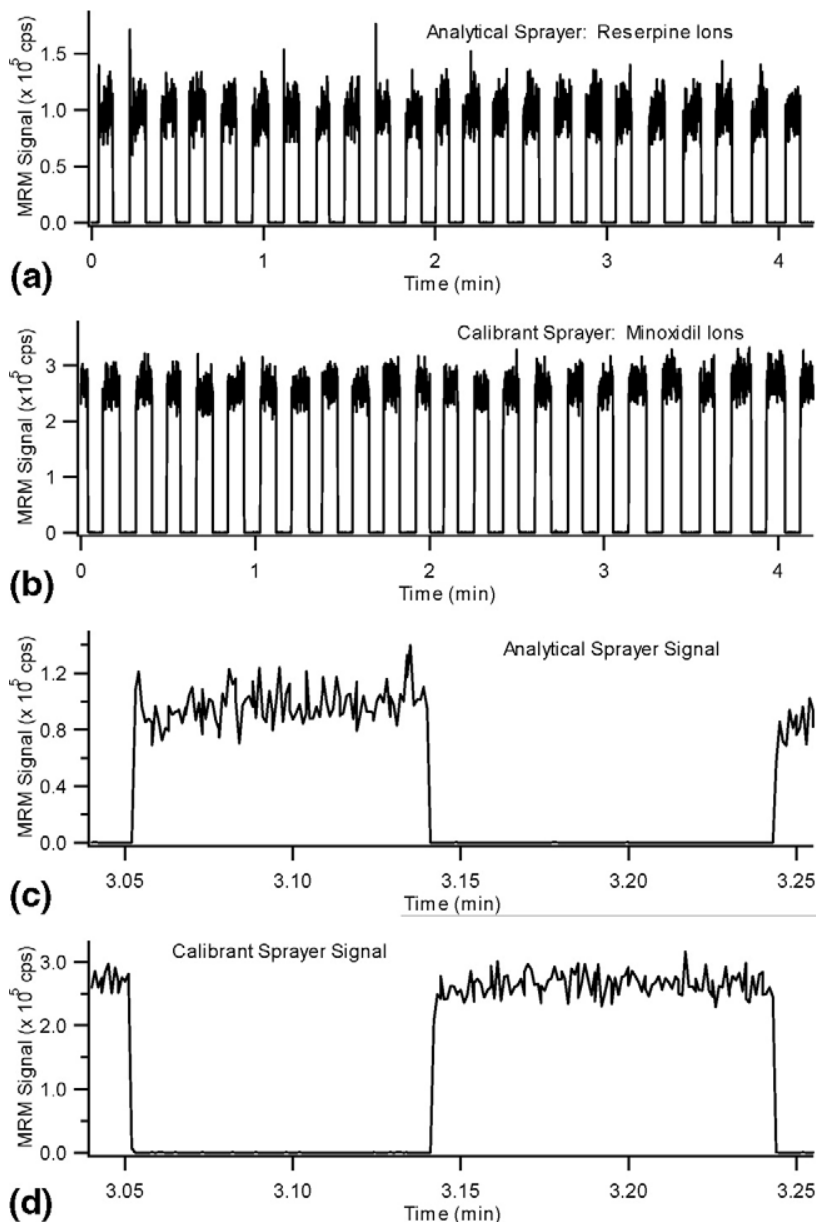

Figure 3. MRM data showing the speed of toggling the calibrant and analytical signals on and off, and the reproducibility of returning the ion current to the original levels after turning off. The analytical spray was comprised of a reserpine standard and the calibrant spray was comprised of minoxidil. The MRM transitions were the same as those used for Figure 2, and the dwell period was $20 \mathrm{~ms}$. (a) MRM signal for reserpine ions generated by the analytical sprayer. (b) MRM signal for minoxidil ions generated by the calibrant sprayer. (c) Expanded view along the time axis for the MRM signal for reserpine ions generated by the analytical sprayer. (d) Expanded view along the time axis for the MRM signal for minoxidil ions generated by the calibrant sprayer.

inside the curtain region. Under these conditions, the loss of ions through the aperture and in the transporting gas flow to the mass spectrometer inlet lowered the measured signals by $\sim 15$ to $50 \mathrm{X}$ relative to when the sprayer was placed in the optimal sampling region for a nanoflow electrospray device, directly in front of the mass spectrometer inlet. This sampling efficiency is similar to what is observed for a pneumatically assisted electrospray source operating at $500 \mu \mathrm{L} / \mathrm{min}$ [7], providing more than adequate sensitivity for calibration purposes.

Figure 3 demonstrates both the speed of toggling the signals and the ability to return the signals to original levels before and after several cycles. Typically, signals could be eliminated within 45 to $100 \mathrm{~ms}$ and returned to within $2.5 \%$ of the original level in a similar time frame.
The ion current stability was similar for both sources and the same as one would achieve with a single sprayer system without a calibrant channel. Similar data were also generated with calibrants that produce multiply charged ions (data not shown). Multiply charged ions experience a greater repelling force than singly charged ions at the mass spectrometer inlet when the analytical sprayer voltage is high so there was no reason to expect otherwise.

All of the preceding data demonstrate operation of the system for external calibration purposes, that is, the calibrant ions and analyte ions are never present within the same spectrum. Internal calibration can be accomplished with the same physical configuration but applying the voltages differently to allow for analyte and calibrant ions to be present in the same spectrum. With either the calibrant sprayer voltage off or at a negative potential, the neutral or negatively charged droplets entrained in the transporting gas will not be repelled by the field between the analytical sprayer and inlet aperture but instead will mix with the droplets from the analytical sprayer and create ions as a result.

When the calibrant nebulizer has the voltage off, neutral droplets and calibrant molecules are introduced into the transport gas. The neutral droplets that migrate in front of the inlet aperture collide with the charged droplets from the analytical sprayer, thereby picking up charge and, upon partial evaporation, emit ions by ion evaporation. This mechanism is similar to previous work involving fused droplets [8-11]. Neutral molecules in this region can also become charged after incorporation into droplets, or by gas-phase charge-transfer. However, unlike these previous examples in the literature, applying a negative potential to the calibrant sprayer introduces negatively charged droplets into the transport gas, which are electrostatically attracted to the positive droplets from the analytical sprayer (or vice versa). The calibrant spray is transported $\sim 5 \mathrm{~cm}$ through the heated curtain chamber before encountering the analytical spray at the sampling aperture. Therefore, the calibrant spray has undergone substantial desolvation and more droplet fissions to yield smaller droplets with less net charge. As operated here the small negative droplets are incorporated into the larger positive droplets from the analytical electrospray and reverse polarity as a result of their interaction to emit positive ions. Figure 4 demonstrates all modes of operations thus far discussed. Figure $4 a$ is a spectrum showing the presence of the calibrant ions (safranin orange) under conditions when neutral drops are introduced into the transport gas. Figure $4 \mathrm{~b}$ also shows the calibrant ions under conditions where negative droplets are introduced into the transport gas. This mode is more efficient at producing positive ions than introducing neutral droplets, presumably because the electrostatic attraction improves the efficiency of the droplet interactions. Experiments with other calibrant molecules such as aztreonam, cyclo- 


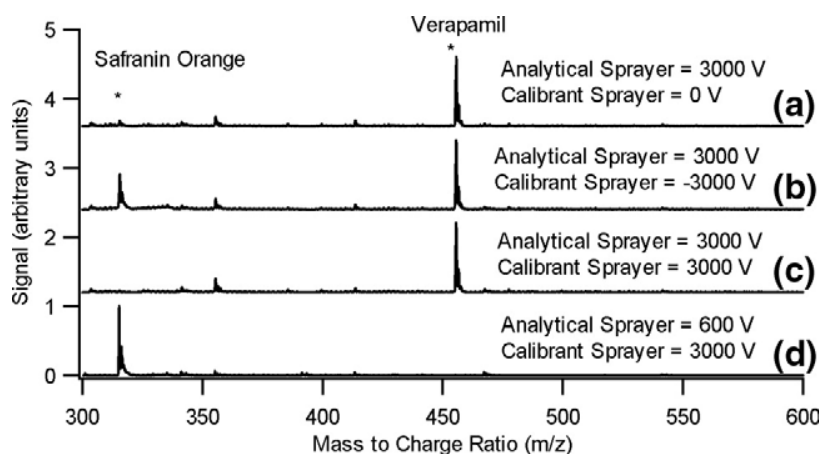

Figure 4. Full scan spectra generated with samples of verapamil in the analytical sprayer and safranin orange in the calibrant sprayer showing means for external calibration as well as internal calibration as the potential applied to the analytical and calibrant sprayers were varied. The potentials applied to the analytical sprayer and calibrant sprayer were $3000 \mathrm{~V}$ and $0 \mathrm{~V}(\mathbf{a}), 3000 \mathrm{~V}$ and $-3000 \mathrm{~V}(\mathbf{b}), 3000 \mathrm{~V}$ and $3000 \mathrm{~V} \mathrm{(c)}$, and $600 \mathrm{~V}$ and $3000 \mathrm{~V}(\mathrm{~d})$, respectively. The solvent flow rates were $500 \mathrm{~nL} / \mathrm{min}$ for the analytical sprayer and $3 \mu \mathrm{L} / \mathrm{min}$ for the calibrant sprayer.

sporine, succinyl choline, steroids, and peptides also showed improvements of $\sim 3$ to $5 \mathrm{X}$ when the calibrant sprayer was operated as a negative mode electrospray as opposed to a nebulizer. When operating in this fashion, calibrant signals improved as the heater temperature was reduced (more penetration of solvent into the curtain chamber), and as the analytical sprayer was positioned farther from the sampling inlet (more interaction time for the analytical spray with the droplets in the counter-current gas flow). Figure $4 \mathrm{c}$ demonstrates, with the same physical apparatus, the external calibration mode where both the calibrant and analytical sprayers are at high potentials, thus turning the calibrant signal off. Figure $4 \mathrm{~d}$ shows the external calibration mode with the analyte signal turned off and calibrant signal on by lowering the analyte sprayer voltage to the same potential as the mass spectrometer inlet. Although not demonstrated here, with this mode of operation it is possible to introduce opposite polarity ions to the mass spectrometer from the two different sources that are sufficiently removed from each other that no detrimental field effects can occur.

This general approach can be used to interface ion sources that produce ions by different ionization mechanisms, such as MALDI and electrospray. Figure $1 \mathrm{~b}$ shows a schematic of a sampling interface incorporating an atmospheric pressure MALDI (AP MALDI) source and a nanospray source located outside of the MALDI housing. The MALDI sample plate is located directly in front of the sampling inlet of a heated laminar flow chamber, with a chamber downstream, designed to direct a counter-current gas flow to the front of the sampling inlet [5]. The physical size of the MALDI sample plate makes it difficult to position an electrospray emitter in close proximity to the sampling inlet. However, as shown in Figure 1b, a nanoflow electrospray emitter can be located out- side the MALDI source housing, with a transfer tube inserted through the curtain plate to provide a conduit for transporting ions from the electrospray source. A nanoflow sprayer is positioned immediately in front of the entrance aperture of the transfer tube such that the combined effects of space charge and high nebulizer gas flows will transport ions through the transfer tube and into the curtain chamber. Ions within the curtain chamber are carried to the sampling inlet by a combination of gas flows and fields, in the same manner as previously described for dual electrospray sources.

Figure 5 shows data generated operating in this fashion, using a $10 \mathrm{~Hz}$ nitrogen laser with the $\mathrm{AP}$ MALDI source. The laser was left running for the entire experiment, desorbing sample from the MALDI plate. The polarity was switched over the course of these experiments with potentials of $620 \mathrm{~V}$ and $-620 \mathrm{~V}$ applied to the curtain plate and heated inlet when operating in the positive and negative ion modes, respectively. A potential of $-2500 \mathrm{~V}$ was continuously applied to the calibration sprayer while the potential applied to the MALDI sample plate was varied between $2500 \mathrm{~V}$ (positive mode) and $-620 \mathrm{~V}$ (negative mode). The nebulizer gas on the calibrant sprayer was $\sim 3.5$ $\mathrm{L} / \mathrm{min}$ directed into the $7 \mathrm{~cm}$ long transfer tube (i.d. $=$ $2.4 \mathrm{~mm}$ ). The samples were $\sim 200 \mathrm{fmol}$ each of three peptides (angiotensin I, bradykinin, and angiotensin II) deposited onto the sample plate, and $\sim 1 \mathrm{ng} / \mu \mathrm{L}$ taurocholic acid sprayed from the calibrant source. Figure $5 \mathrm{a}$ shows the MALDI spectrum of the three peptides. The MALDI plate potential was then lowered to the curtain plate potential and the instrument polarity was switched to negative ion mode over approximately the next $700 \mathrm{~ms}$ to generate the electrosprayed calibrant ion spectrum shown in Figure $5 \mathrm{~b}$ with no trace of the

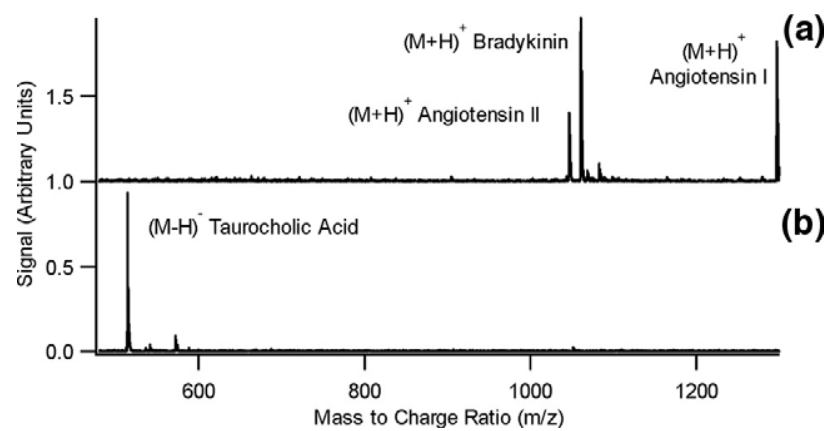

Figure 5. Scan data generated with a three-peptide mixture deposited on a MALDI plate and a sample of taurocholic acid sprayed through an additional nanoflow electrospray source, showing average single scan data in each case. Approximately 200 fmol of each peptide was deposited on the plate, and a small fraction of this was consumed over the course of this experiment. The data were acquired while switching polarities to observe signals corresponding to the protonated peptides (+ve mode) as well as deprotonated taurocholic acid (-ve mode). The potential applied to the nanoflow electrospray source was fixed at $-3000 \mathrm{~V}$ throughout. The target plate potential varied from $2500 \mathrm{~V}$ (a) to $-620 \mathrm{~V}(\mathbf{b})$ 
MALDI ions. These data demonstrate that it is possible to operate different ion sources of opposite polarity when using a mass spectrometer capable of rapid polarity switching.

\section{Conclusions}

This article describes the use of laminar gas flows to transport ions emanating from a variety of independently controlled ion sources to the inlet aperture of an electrospray ion source. The ion beam from these ion sources, positioned substantially remote from the electrospray source, is extremely stable and can be toggled on and off in less than 100 milliseconds. Ions of the same and different polarities can be generated and sampled into the mass spectrometer in rapid succession or simultaneously offering a possible means for mass calibration on demand or means of accumulating ions of different polarity on which to conduct ion-ion reactions.

\section{Acknowledgments}

The authors thank Deolinda Fernandes for preparing all of the samples used in this work. The authors also thank Cindy Schneider for aiding in the preparation of the figures.

\section{References}

1. Breyer, M.; Hucke, K.; Gaefke, D.; Schmeer, K. High Accuracy Mass Measurement by LC-TOF-MS without Lock-Spray. Poster Number 169. Proceedings of the 21st Symposium on LC/MS; Montreux, Switzerland, November, 2004.

2. Schneider, B. B.; Covey, T. R. Molecular Ionization. In The Encyclopedia of Mass Spectrometry, Vol. 6, Gross, M.; Caprioli R., Eds.; Elsevier Publishing Ltd.: Oxford, U.K., 2007, pp 456-467.

3. Schneider, B. B.; Baranov, V. K.; Javaheri, H.; Covey, T. R. Particle Discriminator Interface for Nanoflow ESI-MS. J. Am. Soc. Mass Spectrom. 2003, 14, 1236.

4. Schneider, B. B.; Guo, X.; Fell, L. M.; Covey, T. R. Stable Gradient Nanoflow LC-MS. J. Am. Soc. Mass Spectrom. 2005, 16, 1545-1551.

5. Schneider, B. B.; Lock, C.; Covey, T. R. AP and Vacuum MALDI on a QqLIT Instrument. J. Am. Soc. Mass Spectrom. 2005, 16, 176-182.

6. Rulison, A. J.; Flagan, R. C. Scaleup of Electrospray Atomization Using Linear Arrays of Taylor Cones. Rev. Sci. Instrum. 1993, 64, 683-686.

7. Covey, T. R., Schneider, B. B., Javaheri, H., Leblanc, J. C. Y., Ivosev, G., Corr, J. J., Kovarik, P. ESI, APCI, and MALDI. A Comparison of the Central Analytical Figures of Merit: Sensitivity, Reproducibility, and Speed. Electrospray and MALDI Mass Spectrometry: Fundamentals, Instrumentation, Practicalities, and Applications; Cole, R., Ed.; Wiley InterScience: Indianapolis, IN

8. Shiea, J.; Chang, D. Y.; Lin, C. H.; Jiang, S. J. Generating Multiply Charged Protein Ions by Ultrasonic Nebulization/Multiple ChannelElectrospray Ionization Mass Spectrometry. Anal. Chem. 2001, 73, 49834987.

9. Chang, D. Y.; Lee, C. C.; Shiea, J. Detecting Large Biomolecules from High-Salt Solutions by Fused-Droplet Electrospray Ionization Mass Spectrometry. Anal. Chem. 2002, 74, 2465-2469.

10. Shieh, I. F.; Lee, C. Y.; Shiea, J. Eliminating the Interferences from TRIS Buffer and SDS in Protein Analysis by Fused-Droplet Electrospray Ionization Mass Spectrometry. J. Proteome Res. 2005, 4, 606-612.

11. Chen, H.; Venter, A.; Cooks, R. G. Extractive Electrospray Ionization for Direct Analysis of Undiluted Urine, Milk, and Other Complex Mixtures without Sample Preparation. Chem. Commun. 2006, 19, 2042-2044. 